\title{
Supernova Science Center
}

\author{
Grant DE-FC02-01ER41176
}

\section{Final Report July 15, 2001 - July 15, 2007}

\section{Introduction}

The Supernova Science Center (SNSC) was founded in 2001 to carry out theoertical and computational research leading to a better understanding of supernovae and related transients. The SNSC, a four-institutional collaboration, included scientists from LANL, LLNL, the University of Arizona (UA), and the University of California at Santa Cruz (UCSC). Intitially, the SNSC was funded for three years of operation, but in 2004 an opportunity was provided to submit a renewal proposal for two years. That proposal was funded and subsequently, at UCSC, a one year no-cost extension was granted. The total opeartional time of the SNSC was thus July 15, 2001 - July 15, 2007. This document summarizes the research and findings of the SNSC and provides a cummulative publication list.

Also attached, as scidac04.pdf, is the renewal proposal that was submitted in February, 2004. This describes, in considerable detail, progress at all four member institutions of the SNSC from July 15, 2001 up until the time of the proposal, and lists 96 publications attributable to the grant during its first 30 months. This present document thus focuses of accomplishments from February, 2004 through July, 2007, and provides a cummulative publication list. Many of the same people and science topics supported by the SNSC were also funded in a new SciDAC 2 grant that commenced September 15, 2006 ("Computational Astrophysics Consortium 3: Supernovae and Gamma-Ray Bursts"; DE-FC02-06ER41438) and there was some overlap between the two grants in 2007. Many of our scientific accomplishments under SciDAC 1 formed the basis for this second proposal, but the SciDAC 2 grant is larger in scientific scope, includes more people and has greater funding. Some of our accomplishments under SciDAC 1 were also discussed in that proposal - but a copy is not attached.

\section{Research Highlights}

Some of the major accomplishments of the SNSC during its six years of operation were:

- Core-Collapse Supernovae - Early in the grant, SNSC scientists at LANL carried out the first 3D, full star simulation of core collapse and explosion. Later, at both UA and 
LANL, they also carried out simulations in 2D with multi-group neutrino transport of the collapse, bounce, and early post-bounce evolution. A new supernova mechanism was discovered powered by neutron star vibrations. Gravitational radiation signatures were also computed. Late in the grant, the first 2D models with neutrinos, rotation and magnetohydrodynamics were calculated at Arizona (LANL and Arizona).

- Gamma-Ray Bursts - SNSC scientists at UCSC developed and applied a 3D adaptive mesh relativistic hydro-code to the production of gamma-ray bursts in massive stars. This was a breakthrough in our understanding of GRBs, showing, for example, that bursts had to originate from compact stars and not giants. The calculation was featured in many magazines and press conferences including the jarch, 2007 issue of National Geographic. The eruption of the jet from the surface produced a cocoon of mildly relativistic ejecta that should give rise to observable hard x-ray transients off axis. These may have been observed (UCSC).

- Flames and Explosions in Type Ia Supernovae - Working with scientists at the SciDAC-supported Code Center at LBNL, we carried out simulations of nuclear burning in Type Ia supernovae on all scales, ranging from well-resolved simulations of the flame (less than $1 \mathrm{~cm}$ ), to full-star models for the supernova $\left(10^{9} \mathrm{~cm}\right)$. We examined the physics of the transition to "distributed burning" as turbulence tears the flame, setting the stage for a possible transition to detonation at low density. Our three-dimensional simulations proved, for the first time, that the turbulence generated by the Rayleigh-Taylor instability in a Ta supernova was indeed Kolmogorov-like and became isotropic after approximately one decade in turnover length scale. Other calculations of presupernova evolution showed that ignition would happen in the context of a dipole-like circulation and, consequently, ignition would be one-sided and off-center. We explored the consequences of such ignition in full star 2D and 3D calculations in collaboration with the Munich group (UCSC, LBNL, and the MPA).

- Radiation transport in Supernovae - Late in the grant, we explored the physical reason why a width-luminosity relation exists in Type Ia supernovae, discovering that it is largely due to a readjustment of the color at which the supernova radiates. This in turn reflects the transition in the dominant ionization of iron-group speces from doubly ionized to singly-ionized. We also carried out studies of the Type Ia supernova light curve for a variety of explosion models to better understand the influence of parameters other than the ${ }^{56} \mathrm{Ni}$ abyndance. (UCSC and Johns Hopkins)

- Nucleosynthesis - The synthesis of the elements was explored for a large variety of explosions ranging from a putative first generation of very massive stars, to modern Type II supernovae with solar metallicity. Nucleosyntheisis in neutrino winds and gamma-ray burst accretion disks was also explored. We were able to produce the solar abundance set using a relatively straightforward grid of parameterized explosion 
models and we co-discovered a new nucleosynthesis process, the neutrino boosted rp-process in the neutrino wind. State-of-the-art nuclear reaction data bases were necessary for these studies and were prepared by the LLNL team working with nuclear scientists from all over the world. (LANL, LLNL, UCSC, Arizona)

- Code Development - We worked with the SciDAC Institute at LBNL, especially with John Bell and Ann Almgren, to develop a new set of tools for studying supernova physics at low Mach number (especially stellar convection, Type Ia supernova ignition, and low Mach Number flame physics). Two codes, SNe and a preliminary version of MAESTRO, were fielded. Later under SCiDAC 2, MAESTRO was completed and in 2007 is being applied to problems in the field. At UCSC, Gary Glatzmaier's 3D anelastic hydrodynamics code was rewritten and applied to Type Ia supernova ignition and other problems in stellar convection. At the University of Arizona, the spherically symmetric code SESAME was developed to solve the full Boltzmann equation using the Feautrier and tangent-ray techniques and an accelerated $\Lambda$ iteration. This code is multi-group, mutli-angle, and multi-species. A 2D code, VULCAN, was developed at Arizona that incorporated multi-group neutrino transport, rotation, and magnetohydrodynamics. This was applied in 2007 to do the first 2D calculations of a rotating, MHD supernova model. Zingale (UCSC) sped up the gravity solver in FLASH by two orders of magnitude and implemented spherical coordinates and logarithmic mesh in this adaptive mesh code. A 3D smoothed particle hydrodynamics code, SNSPH, was written at LANL and adapted to the core-collapse supernova problem. The LANL internal unclassified version of RAGE was also adapted to supernova studies (Arizona, UCSC, LBNL, LANL).

- 3D Monte Carlo - At LANL, studies of radiation transport were carried out for symmetric and asymmetric supernovae using an implicit 3D Monte Carlo code. They found significant differences in the velocity distribution of radioactive nickel between the symmetric and asymmetric explosion models. The effects of this spatial distribution change are reflected in the overall high energy spectrum, as well as in the individual gamma-ray line profiles. The asymmetric models are much brighter at all angles, in agreement with observations (LANL).

- X-Ray Bursts - SNSC scientists carried out the world's best simulations of Type I x-ray bursts on neutron stars. The nuclear physics of these explosions is a primary science goal of RIA. (LANL, UCSC, LLNL)

Additional research topics and progress can be discerned by reading the titles of the over 100 refereed publications given in the bibliography. 


\section{Research by Institution}

\subsection{Research at UCSC}

At UCSC, research focused upon: a) the presupernova evolution of massive stars; b) studies of nucleosynthesis in massive stars and supernovae; c) thermonuclear (Type Ia and pair-instability) supernovae; d) gamma-ray bursts; and e) x-ray bursts. UCSC did not work on core collapse per se, but on essentially all other aspects of supernova science.

In collaboration with Alex Heger, the first part of a survey of nucleosynthesis in massive stars was completed including a large range of masses and two metallicities (solar and zero). Nucleosynthesis was calculated for approximately 1300 model supernovae using the best available nuclear physics and reaction networks of up to 2000 nuclides. The stars

were evolved to the presupernova state with about 1000 zones each and then exploded using pistons. Mixing and fall were included in a parametric way and the nucleosynthesis of all isotopes from helium through lead determined (except for the $r$-process whose site remains poorly understood).

To the extent possible in a 1D code, Heger and Woosley also studied the transport and effects of angular momentum and magnetic fields in massive stellar evolution. The principal goal was an accurate description of the rotation rate as a function of radius in the presupernova star when all known mechanisms for angular momentum transport are considered, including the effects of magnetic torques. Estimated magnetic field strengths come from assuming axial field winding by differential rotation and radial field generation by the interchange instability according to an approximation given by Henk Spruit in 2001. Without magnetic torques, we obtained presupernova stars whose iron cores would collapse to neutron stars rotating at break up. This would make rotation an essential ingredient in any realistic study of the explosion mechanism. With torques the periods of neutron stars vary with the progenitor star's mass, but were of the order of $7 \mathrm{~ms}$. In these models rotation might (barely) be negligible in the explosion. Obviously these results are uncertain, but their potential importance made the calculations well worth attempting.

A major result was our work on the presupernova evolution of white dwarfs leading up to their ignition and explosion, especially the last two minutes of the life of a carbon-oxygen white dwarf just before it explodes. Convection goes on in the cores of these stars for several hundred years following carbon ignition until the burning rate finally becomes so violent that localized flame sheets are formed. The ensuing deflagration explodes the star in about a second. It is known that the outcome of the explosion, including the supernova energy and light curve, is sensitive to where and how often the flame is ignited. Three approaches were employed - an analytic model based upon mixing-length theory, a 3D anelastic numerical simulation using Glatzmaier's code, and a 1D simulation using the "One-Dimensional Turbulence" algorithm developed by SNSC collaborators Scott Wunsch and Alan Kerstein 
at Sandia Laboratory, Livermore. We found that the supernova was ignited off-center and on one side. However, just where and how often is inherently unpredictable (chaotic). This means that there will always be some degree of unpredictable variation in the properties of Type Ia supernova, a fact that ultimately limits how far they can be used as standard candles.

A very productive collaboration was established with scientists in the LBNL ISIC in integrated software resulting in the application to supernovae of the low-Mach number code developed at LBNL to study ordinary terrestrial combustion. Supernova physics was implemented in the LBNL code and the resultant code used to study the Landau-Darrieus and Rayleigh-Taylor instabilities in Type Ia supernovae, especially in the region where the flame becomes thick and a transition to detonation, possible. Our results showed clearly the transition from the flamelet regime to turbulent burning. Properties of the turbulence were established showing that it had a Kolmogorov power spectrum and quickly became isotropic in the vicinity of the flame front. This productive collaboration continues well into the SciDAC 2 era.

Towards the end of this grant and on into the beginning of SciDAC 2, Woosley and postdoc Fritz Röpke carried out 3D full-star modeling of Type Ia supernovae using the Munich group's code. First the effect of igniting the supernova in multiple locations off-center was explored. Not surprisingly, enough points ignited far enough off center gave vigorous explosions. In late 2006 and early 2007 though, we began to explore the possibly more realistic case of single point ignition off center. About the same time the Chicago FLASH Center published calculations claiming a robust explosion resulting from these initial conditions - the so called "Gravitationally-Confined Detonation Model" (GCD). Matter breaking out of the white dwarf formed waves that traveled around the white dwarf colliding on the other side and initiating a detonation. We found a similar occurrence in $2 \mathrm{D}$ but not in $3 \mathrm{D}$. The difference was traced to the treatment of the flame speed in the ascending part of the initial burning. With less burning, Chicago expanded the white dwarf less and got a more violent collision on the far side. We got more expansion because of a (we feel) superior treatment of the subgrid physics. The controversy and calculations are still continuing in late 2007.

On another subject, Zhang and Woosley simulated, in 3D, the propagation of relativistic jets through massive stars. Their results were highlighted on the cover of the overall SciDAC 1 five year progress report (by Jon Bashor), and in numerous other publications, popular and otherwise. These jets are created deep inside the star when a black hole formed following iron core collapse accretes the rest of the inner star through a disk. The jet both explodes the star as a "hypernova" and by internal collisions outside the star makes a gamma-ray burst. The burst is only seen by observers coalligned with the jet. During the last six months we have been especially examining the break out of this jet through the stellar surface. It turns out that the mildly relativistic but highly pressurized 
"cocoon" of gas surrounding the jet experiences its own explosion as the jet breaks out. This sends relativistic material to all angles and suggests that even observers that do not see a gamma-ray burst will still see a high energy display, possibly a hard x-ray flash. Such phenomena have been observed and have thus far eluded explanation. These calculations were 2D. During the last two months we have made the transition to 3D. The general characteristics of the $2 \mathrm{D}$ results are preserved. There is still a focused jet at break out and a cocoon explosion. But the jets are much less symmetric. Small (1\%) perturbations in

energy input from one side to the other grow in a non-linear way and give quite asymmetric jets at break out.

SNSC scientists at UCSC and scientists from JINA also carried out the world's foremost simulations to date of Type I x-ray bursts. Although not supernovae, XRBs are astrophysical thermonuclear explosions that develop on the surfaces of accreting neutron stars. They can be treated with the same codes and techniques developed to study supernovae and there exists a wealth of astronomical data to which one may compare the results. The challenge here was coupling large nuclear reaction networks to stellar models in tight hydrostatic equilibrium but where a convective runaway is happening. The energy generation depends on flows among hundreds of nuclei near the proton-drip line and simple approximations are inadequate. An additional complication is that the heat and ashes from one burst affects the critical mass for and properties of the next burst. We computed bursts for two compositions and two different accretion rates for a total of between 6 and 14 repeated flashes in each case. We found that the first burst is more violent than subsequent bursts because it occurs on a relatively cold inert substrate. Only these most violent first bursts produce elements as heavy as tin. Others go barely beyond the iron group. After the first burst, the bursts became self-similar. The critical mass was less in low metallicity cases than expected because the ashes of the previous burst play a role in ignition.

\subsection{The University of Arizona}

The University of Arizona was one of the two SNSC sites specializing in core-collapse physics and neutrino-powered supernovae. The focus at UA was on creating, optimizing, and running grid-based codes to simulate the collapse and explosion of core-collapse supernova in 1D, 2D, and 3D. The one-dimensional code, SESAME, was written for the problem. SESAME simulates the hydrodynamical evolution and neutrino radiation fields during stellar core collapse and achieves high angular and energy resolution. The full Boltzmann neutrino transport equations were solved via a variable Eddington factor technique and the tangent-ray method. The hydrodynamics was Lagrangian and explicit, but the transport was fully implicit. Multi-energy-group, multi-angle, and multi-species neutrino transport was included. The various flavors of neutrino were handled separately and coupling to matter was facilitated with accelerated $\Lambda$ iteration (ALI). This code was be 
our spherically-symmetric benchmark for explorations using other codes in 2D, and in the future, 3D. Despite the codes prowess, no realistic 1D model was ever found that exploded robustly. Whatever core-collapse supernovae are, they must involve multi-dimensional effects - convection, asymmetric accretion, rotation, and/or magnetic fields.

The UA team also programmed a 2D variant, called VULCAN/2D, a multi-group, multi-angle $\left(S_{N}\right)$, moving-grid, Arbitrary Lagrangian-Eulerian (ALE) scheme that incorporated rotation and magnetic fields (in "2.5-D"). Since this code required the solution of partial differential equations in 1 (time) +2 (space) +1 (energy) +1 (angle) $=5$ dimensions, highly parallel and optimized algorithms were required to achieve useful turnaround times and resolution. For VULCAN/2D, they achieved this by parallelizing in energy groups and using efficient sparse matrix solvers for the angular sector and have achieved near linear speed-ups. They have ported VULCAN to the ORNL and NERSC machines and are now doing test runs. Computer scientists at UA/ACIMS also created a Parallel Programming and Debugging Facility (ADViCE, PAMS) and a Runtime and Execution infrastructure (CORBA, DEVS-DOC) that allowed us a better understanding of and facilitated debugging of the complicated multi-dimensional variants. This system was tested by parallelizing SESAME in neutrino species and energy groups and profiling the results. Near linear speed-ups were been achieved.

Using VULCAN/2D, UA SNSC scientists explored a variety of supernova models with and without magnetic fields and rotation. A new supernova mechanism based upon acoustic energy transport was discovered. The standing accretion shock instability leads to a situation where accretion occurs on one side of the neutron star and pounds it like a hammer. The neutron star rings like a bell sending sound wave out into the surrounding medium where they steepen into shocks. Robust explosions are obtained for all models studied to date. When rotation and magnetic fields were included in a rapidly rotating model, jet formation was observed, also accompanied by a supernova. The realization is growing that the supernova "mechanism" may actually be a collection of mechanisms which have variable efficiency for progenitors of different masses. For the lightest supernovae, 10 - 12 solar masses, neutrinos alone may suffice, but for heavier stars some combination of acoustic and MHD transport is likely to be essential.

In addition, Burrows and colleagues incorporated a gravitational wave signature extraction routine into VULCAN/2D and calculated the signatures with realistic equations of state and initial models. They also did simulations with VULCAN/2D of various hydrodynamic instabilities during the infall phase of supernova dynamics, as well as the post-bounce phase. Unique to this study was the inclusion of the effects of core motion when a full gravity solver (recently updated in VULCAN) was used.

All of these studies are continuing under the SciDAC 2 grant. 


\subsection{Research at LANL}

LANL was the other SNSC site specializing in core collapse physics and neutrinopowered supernovae. They adopted a different approach to 2D- and 3D-supernova hydrodynamics that emphasized the smooth particle hydrodynamics approach pioneered by Marc Herant, Chris Fryer, and their colleagues. They also studied radiation transport Monte Carlo and multi-group - and gravitational radiation from asymmetric explosions and are currently explored the use of the (unclassified) RAGE code developed at LANL as a supernova platform.

RAGE is an AMR code developed within the X-2 Division at LANL. It is an unclassified code that uses 1, 2, or 3D adaptive mesh refinement on an Eulerian grid. Radiation transport is included in the grey diffusion approximation. Analytic equation of state and opacity modules can be used. Energy sources can be specified and the code is parallel optimized. Gravity was implemented in the code (using a tree scheme) and the result was optimized to run on parallel machines. Fryer and his colleagues also added a number of equations of state to the code, including the Swesty-Lattimer version. This code was used to study mixing in supernovae. Here, RAGE has a big advantage in that it has several subgrid turbulence algorithms that have been tested against experiment.

Asymmetric explosions were simulated in a 3D code by using pistons whose velocity varied with angle (twice as fast at the pole than in the equator). Calculations were run as long as one year to study mixing and the effect on gamma-ray transport, the latter calculated using a 3D implicit Monte-Carlo code. Fryer and Hungerford found that, for explosions of the same energy, asymmetric explosions mix more and have emit brighter gamma-ray line fluxes earlier. It looks likely that a factor of 2 asymmetry will be enough to explain observations of SN 1987A where the gamma-ray lines appeared earlier and were broader than expected. Enhanced emission is found even along the weaker explosion axis because the photons emitted in the material along the strong explosion axis can easily escape both out along the pole and also along trajectories perpendicular to their motion. LANL followed up on these 3D explosion simulations by mapping 2D collapse simulations of rotating stars (hence an asymmetric explosion) $1.5 \mathrm{~s}$ after bounce into the 3D explosion code. These simulations were then run to late times with a small nuclear burning network to get reasonably accurate energetics.

LANL SNSC scientists also modeled the first 3-dimensional asymmetric collapse simulations caused by mixing and oscillations in the progenitor star. They found that in order to get neutron star kicks the initial perturbation must be much larger than those predicted in the 2D simulations of Burrows and Hayes. Even with these large perturbations, the resultant explosion was much more symmetric than had been believed in 2D simulations. However, large neutrino asymmetries $(>1 \%)$ produced sizable kicks and strong gravitational wave signals. 
With Milne, Hungerford and Fryer compared the results from gamma-ray line transport codes currently being used by the astrophysics community and their detailed Monte-Carlo results. The comparison revealed major errors in some of these codes. In particular the errors found in the two principal codes used by the community to calculate supernova light curves call into question the accuracy of many published papers.

\subsection{Research at LLNL}

LLNL was the lead SNSC institution for assembling, calculating, and maintaining the nuclear data library needed for studies of supernovae and nucleosynthesis. Research at LLNL focused heavily upon: 1) standardized reaction rate libraries for nuclear astrophysics; 2) nucleosynthesis associated with gamma ray bursts; and 3) nucleosynthesis in the neutrino-powered wind.

Rob Hoffman compiled an up-to-date tabulation of experimental and theoretical data and Pruet and Fuller extended calculations of weak interaction rates to heavy nuclei $(\mathrm{A} \lesssim 110)$. These weak rates were needed for simulations of late-time presupernova stellar evolution as well as for simulations of x-ray bursts.

With the discovery of supernovae accompanying gamma-ray bursts, people became interested in determining what nucleosynthesis might characterize these unusual events. A number of observations suggested that GRB events produced radioactive ${ }^{56} \mathrm{Ni}$ in substantial amounts. Pruet, Woosley, and Hoffman showed that these observations have important implications for conditions in the central engine as well as for the synthesis of the r-process elements. This happens because there is an intimate connection between the composition (neutron to proton ratio) and properties (mass accretion rate and viscosity) of the black hole accretion disk widely thought to power the GRB. A neutron to proton ratio smaller than unity, needed for efficient synthesis of ${ }^{56} \mathrm{Ni}$, can only occur in accretion disks that are accreting at a rate less than about 1/10 of a solar mass per second. Alternatively, if just one percent of the matter ejected from the disk is ejected while the accretion rate is larger than $2 / 10$ of a solar mass per second, then this could account for the observed $r$-process elements. As well as constituting a force to be reckoned with in terms of galactic chemical evolution, GRBs should also generically produce substantial local overabundances of deuterium.

In addition to investigating the nucleosynthetic signature of the GRB environment, the LLNL group also studied what the photons and neutrinos from GRBs can tell us. Because neutrons are uncharged and essentially only couple to the high entropy GRB fireball via strong interactions, their dynamics can differ dramatically from the dynamics of the rest of the fireball. Pruet and Dalal calculated implications of these dynamics for observed GRB light curves and identified a subset of bursts that show evidence for a neutron rich outflow. There may also be a neutrino signature of the composition and origin of GRBs. Pruet 
showed that if "failed supernovae" (collapsars) are responsible for observed GRBs, then inelastic neutron-nucleon collisions will produce a multi-GeV neutrino burst that precedes the main GRB photon emission by about 10 seconds. These neutrinos may be detectable in next generation neutrino telescopes and their observation would solidify the connection between SNe and GRBs.

In 2005 and 2006, SNSC scientists at LLNL and UCSC explored what happens in neutrino-powered wind of a young neutron star. One of the outstanding unsolved riddles of nuclear astrophysics is the origin of the so-called p-process nuclei from $\mathrm{A}=92$ to 126 . Both the lighter and heavier p-process nuclei are adequately produced in the neon and oxygen shells of ordinary Type II supernovae, but the origin of these intermediate isotopes, especially 92,94Mo and 96,98Ru, has long been mysterious. Pruet et al. considered such early times that the neutrino-powered wind still contained a proton excess (because the rates for electron neutrinos and positron captures on neutrons are faster than those for the inverse captures on protons). They also included the possibility that-in addition to the protons, $\alpha$-particles, and heavy seed - a small flux of neutrons is maintained by the reaction neutrinos capturing on free protons. This flux of neutrons is critical in bridging the long waiting points along the path of the rp-process by $(n, p)$ reactions. Using the unmodified ejecta histories from a recent two-dimensional supernova model by Janka and coworkers, they found synthesis of p-rich nuclei up to $102 \mathrm{Pd}$, although the calculations still did show efficient production of $92 \mathrm{Mo}$. If the entropy of these ejecta is increased by a factor of 2 , the synthesis extends to 120Te. Still larger increases in entropy, which might reflect the

role of magnetic fields or vibrational energy input neglected in the hydrodynamical model, result in the production of nuclei up to A 170. Elements synthesized in these more extreme outflows included numerous s- and p-process nuclei, and even some r-process nuclei can be synthesized in these proton-rich conditions.

\section{REFERENCES}

\section{Refereed - Cumulative, July 15, 2001 - 2007}

Alexakis, A., Calder, A. C., Heger, A., Brown, E. F., Dursi, L. J., Truran, J. W., Rosner, R., Lamb, D. Q., Timmes, F. X., Fryxell, B., Zingale, M., Ricker, P. M., \& Olson, K. 2004, "On Heavy Element Enrichment in Classical Novae", Astrophys. J. , 602, 931

Almgren, A. S., Bell, J. B., Rendleman, C. A., \& Zingale, M. 2006, Low Mach Number Modeling of Type Ia Supernovae. I. Hydrodynamics" Astrophys. J. , 637, 922 - 936

Almgren, A. S., Bell, J. B., Rendleman, C. A., \& Zingale, M. 2006, "Low Mach Number Modeling of Type Ia Supernovae. II. Energy Evolution", Astrophys. J. , 649, 927 938 
Baraffe, I., Heger, A., and Woosley, S.E. (2004), "Stability of Supernova Ia Progenitors against Radial Oscillations", Astrophys. J. , 615, 378

Bell, J. B., Day, M. S., Rendleman, C. A., Woosley, S. E.\& Zingale, M. A. 2004, "Direct Numerical Simulations of Type Ia Supernovae Flames I: The Landau-Darrieus Instability", Astrophys. J. , 606,1029

Bell, J. B., Day, M. S., Rendleman, C. A., Woosley, S. E.\& Zingale, M. A. 2004, "Direct Numerical Simulations of Type Ia Supernovae Flames II: The Rayleigh-Taylor Instability", Astrophys. J. , 608, 883

Bell, J. B., Day, M. S., Rendleman, C. A., Woosley, S. E.\& Zingale, M. A. 2004, "Adaptive Low Mach Number Simulations of Nuclear Flame Microphysics", J. Comp. Phys.,195, 2,677

Burrows, A., Walder, R., Ott, C. D., \& Livne, E. 2005, "Supernovae, Rotation, and Bipolar Explosions", Nuclear Physics A, 752, 570 - 579

Burrows, A., Livne, E., Dessart, L., Ott, C. D. and Murphy, J., 2006, "A New Mechanism for Core-Collapse Supernova Explosions",Astrophys. J. , 640, 878

Burrows, A., Livne, E., Dessart, L., Ott, C. D., \& Murphy, J. 2006, n Acoustic Mechanism for Core-Collapse Supernova Explosions" New Astronomy Review, 50, 487 - 491

Burrows, A., Reddy, S., \& Thompson, T. A. 2006, "Neutrino Opacities in Nuclear Matter", Nuclear Physics A, 777, 356 - 394

Burrows, A., Livne, E., Dessart, L., Ott, C. D., and Murphy, J., 2007 "Features of the Acoustic Mechanism of Core-Collapse Supernova Explosions", Astrophys. J. , 655, 416

Burrows, A., Dessart, L., Ott, C. D., \& Livne, E. 2007, Multi-Dimensional Explorations in Supernova Theory", Physics Reports, 442, 23 - 37

Calder, A. C., Dursi, L. J., Fryxell, B., Plewa, T., Weirs, V. G., Dupont, T., Robey, H. F., Drake, R. P., Remington, B. A., Dimonte, G., Hayes, J., Stone, J. M., Ricker, P. M., Timmes, F. X., Zingale, M., \& Olson, K. 2004, "Issues with Validating an Astrophysical Simulation Code",Computing in Science and Engineering, vol. 6, no. 5,10

Dessart, L., Burrows, A., Ott, C. D., and Livne, E., 2006 "Multi-Dimensional Radiation/Hydrodynamic Simulations of Protoneutron Star Convection", Astrophys. J. , 645, 534 
Dessart, L., Burrows,A., Ott, C. D., Livne, E., Yoon, S.-Y., and Langer, N., 2006, "Multi-Dimensional Simulations of the Accretion-Induced Collapse of White Dwarfs to Neutron Stars", Astrophys. J. , 644, 1063

Dursi, L. J., Zingale, M., Calder, A. C., Fryxell, B., Timmes, F. X., Vladimirova, N., Rosner, R., Caceres, A., Lamb, D. Q., Olson, K., Ricker, P. M., Riley, K., Siegel, A.,\& Truran, J. W. 2003, "The Response of Astrophysical Thermonuclear Flames to Curvature", Astrophys. J. , 595, 955.

Fryer, C. L., \& Warren, M. S. 2002, "Modeling Core-Collapse in 3 Dimensions", ApJ, 574, L65.

Fryer, C. L. \& Mészaros, P. 2003, "Neutrino-Driven Explosions in GRBs and Hypernovae", ApJL, 588, L25

Fryer, C.L. and Warren, M.S. 2004, "The Collapse of Rotating Massive Stars in 3-Dimensions", ApJ, 601, 391

Fryer, C.L. 2004, "Neutron Star Kicks from Asymmetric Collapse", ApJL, 601, L175 - L178

Fryer, C. L., Holz, D. E., Hughes, S. A. 2004, "Gravitational Waves from Stellar Collapse: Correlations to Explosion Asymmetries", ApJ, 609, 288 - 300

Fryer, C. L. "Core-collapse model effects on nucleosynthesis", 2004, New Astronomy, 48, 13 $-17$

Fryer, C. L., \& Heger, A. 2005, ” Binary Merger Progenitors for Gamma-Ray Bursts and Hypernovae", Astrophys. J. , 623, 302

Fryer, C. L., Hungerford, A. L., \& Timmes, F. X. 2005, "Changing the r-Process Paradigm" Nuclear Physics A, 758, 599 - 602

Fryer, C. L., \& Kusenko, A. 2006, "Effects of Neutrino-driven Kicks on the Supernova Explosion Mechanism", Astrophys. J. Suppl. Ser. , 163, 335

Fryer, C. L., Rockefeller, G., \& Warren, M. S. 2006, "SNSPH: A Parallel Three-dimensional Smoothed Particle Radiation Hydrodynamics Code", Astrophys. J. , 643, 292 - 305

Fryer, C. L., Herwig, F., Hungerford, A., \& Timmes, F. X. 2006, "Supernova Fallback: A Possible Site for the r-Process", Astrophys. J. Lett. , 646, L131 - L134

Fryer, C. L., Rockefeller, G., \& Young, P. A. 2006, "The Environments around Long-Duration Gamma-Ray Burst Progenitors", Astrophys. J. , 647, 1269 - 1285

Fryer, C. L., Young, P. A., \& Hungerford, A. L. 2006, Astrophys. J. , "Explosive Nucleosynthesis from Gamma-Ray Burst and Hypernova Progenitors: Direct Collapse versus Fallback", 650, 1028 - 1047 
Fryer, C. L. 2006, "Fallback in stellar collapse", New Astronomy Review, 50, 492 - 495

García-Senz, D., Bravo, E., Cabezón, R. M., \& Woosley, S. E. 2007, "Constraining Deflagration Models of Type Ia Supernovae Through Intermediate-Mass Elements", Astrophys. J. , 660, $509-515$

Heger, A., Fryer, C., Woosley, S. E., Langer, N., \& Hartmann, D. H. 2003, "How Massive Single Stars End their Life", Astrophys. J. , 591, 288

Heger, A., Woosley, S. E., \& Spruit, H. 2003, "Presupernova Evolution of Rotating Massive Stars

Including Magnetic Fields", Astrophys. J. , 626, 350 - 363

Heger, A., Kolbe, E., Haxton, W. C., Langanke, K., Martinez-Pinedo, G., \& Woosley, S. E. 2003, "Neutrino Nucleosynthesis, Physics Letters B, 606, 258 - 264

Heger, A., Kolbe, E., Haxton, W. C., Langanke, K., Martínez-Pinedo, G., \& Woosley, S. E. 2005, "Neutrino Nucleosynthesis", Physics Letters B, 606, 258 - 264

Hubeny, I., \& Burrows, A. 2007, "A New Algorithm for Two-Dimensional Transport for Astrophysical Simulations. I. General Formulation and Tests for the One-Dimensional Spherical Case" Astrophys. J. , 659, 1458 - 1487

Hungerford, A.L., Fryer, C.L. and Warren, M.S. 2003, "Gamma-Ray Lines from Asymmetric Supernovae", ApJ, 594, 390 - 403

Hungerford, A., Fryer, C.L., \& Rockefeller, 2005, G.M., "Gamma-Rays from Single Lobe Explosions", Astrophys. J. , 635, 487 - 501

Hungerford, A. L., "Radioactive Decay Lines from Asymmetric Supernova Explosions", 2004, New Astronomy, 48, 19 - 24

Hungerford, A. L., Fryer, C. L., Timmes, F. X., \& McGhee, K. 2005, "Nucleosynthetic Signatures of Asymmetric Supernovae - Lessons from 1-dimensional Explosions", Nuclear Physics A, 758, 15

Hwang, et al. 2004, "A Million Secon Chandra View of Cassiopeia A", ApJ, 615, L117 L120

Kuhlen, M., Woosley, S. E., and Glatzmaier, G. 2005, "Carbon Ignition in Type Ia Supernovae", Astrophys. J. , 640, 407 - 416

Livne, E., Burrows, A., Walder, R., Lichtenstadt, I., Thompson, T.A. 2003, "Twodimensional, Time-dependent, Multi-group, Multi-angle Radiation Hydrodynamics Test Simulation in the Core-Collapse Supernova Context," Astrophys. J. , 609, 277 287 
Milne, P., et al., "Unified 1-D Simulations of Gamma-Ray Line Emission from Type Ia Supernovae", 2004, Astrophys. J. , 613, 1101

Murphy, J. W., Burrows, A., \& Heger, A. 2004, "Pulsational Analysis of the Cores of Massive Stars and Its Relevance to Pulsar Kicks", Astrophys. J. , 615, 460 - 474

Nassar, H and 23 others including A. Heger, 2006, The 40Ca(a,g)44Ti Reaction in the Energy Regime of Supernova Nucleosynthesis", Physical Review Letters, 96, 041102

Ott, C.D., Burrows, A., Livne, E., and Walder, R. 2004, "Gravitational Waves from Axisymmetric, Rotating Stellar Core Collapse," ApJ, 600, 834

Ott, C. D., Ou, S., Tohline, J. E., \& Burrows, A. 2005, "One-armed Spiral Instability in a Low-T/-W - Postbounce Supernova Core", Astrophys. J. Lett., 625, L119 - L122

Ott, C. D., Burrows, A., Thompson, T. A., Livne, E., \& Walder, R. 2006, "The Spin Periods and Rotational Profiles of Neutron Stars at Birth", Astrophys. J. Suppl. Ser. , 164, $130-155$

Ott, C. D., Burrows, A., Dessart, L., \& Livne, E. 2006, "A New Mechanism for Gravitational-Wave Emission in Core-Collapse Supernovae", Physical Review Letters, 96, 201102

Ploumans, P., Winckelmans, G.S., Salmon J.K., Leonard A. and Warren, M.S. "Vortex Methods for High-Resolution Simulation of Three-Dimensional Bluff Body Flows; Application to the Sphere at $\mathrm{Re}=300,500$ and 1000", Journal of Computational Physics, 178, 427-463 (2002)

Pruet, J., Woosley, S. E., \& Hoffman, R. D. 2003, "Nucleosynthesis in Gamma Ray Burst Accretion Disks", Astrophys. J. , 586, 1254.

Pruet, J. \& Dalal, N. 2002, "Implications of Neutron Decoupling in Short Gamma Ray Bursts", Astrophys. J. , 573, 770

Pruet, J., Guiles, S., \& Fuller, G. M. 2002, "Light-Element Synthesis in High-Entropy Relativistic Flows Associated with Gamma-Ray Bursts", Astrophys. J. , 580, 368.

Pruet, J., \& Fuller, G. M. 2003, "Estimates of Stellar Weak Interaction Rates for Nuclei in the Mass Range A=65-80", ApJS, 149, 189

Pruet, J. 2003, "Neutrinos from the Propagation of a Relativistic Jet Through a Star", ApJ, 591, 1104.

Pruet, J., Thompson, T., \& Hoffman, R. D. 2004, "Nucleosynthesis in Outflows from the Inner Regions of Collapsars", ApJ, 604, in press. 
Pruet, J., Surman, R. \& McLaughlin, G.C. 2004 "On the Contribution of Gamma Ray Bursts to the Galactic Inventory of Some Intermediate Mass Nuclei", ApJL, 602, L101 - L104

Pruet, J., Woosley, S.E., Buras, R., Janka, H.-T., and Hoffman, R. D. 2005, "Nucleosynthesis in the Hot Convective Bubble in Core-Collapse Supernovae" , Astrophys. J. , 623, $325-336$.

"Nucleosynthesis in Early Supernova Winds. II. The Role of Neutrinos", Pruet, J., Hoffman, R. D., Woosley, S. E., Janka, H.-T., \& Buras, R. 2006, Astrophys. J. , 644, 1028 1039

Rauscher, T., Heger, A,, Hoffman, R. D., \& Woosley, S. E. 2002, "Nucleosynthesis in Massive Stars with Improved Nuclear and Stellar Physics", ApJ., 576, 323.

Robinson, K., Dursi, L. J., Ricker, P. M., Rosner, R., Calder, A. C., Zingale, M., Linde, T., Caceres, A., Fryxell, B., Olson, K., Riley, K., Siegel, A., Truran, J. W., \& Vladimirova, N. 2004, "Morphology of Rising Hydrodynamic and Magneto-hydrodynamic Bubbles from Numerical Simulations", Astrophys. J. , 601, 621

Robinson, K., Dursi, L. J., Ricker, P. M., Rosner, R., Calder, A. C., Zingale, M., Linde, T., Caceres, A., Fryxell, B., Olson, K., Riley, K., Siegel, A., Truran, J. W.,\& Vladimirova, N. 2004, "Morphology of Rising Hydrodynamic and Magneto-hydrodynamic Bubbles from Numerical Simulations", ApJ, 601, 621.

Rockefeller, G., Fryer, C. L., Melia, F., Warren, M. S., "Diffuse X-Rays from the Inner 3 Parsecs of the Galaxy", ApJ, 604, 662 - 670

Rockefeller, G. M., Fryer, C.L., Baganoff, F.K., Melia, F. "The X-ray Ridge Surrounding Sgr A* at the Galactic Center", ApJL, 635, L141 - L144

Rockefeller, G. M., Fryer, C.L., Baganoff, F.K., Melia, F. 2005, "The Sgr B2 Echo of the Galactic Center Supernova Explosion that produced Sgr A East", Astrophys. J. , 638, $786-796$

Rockefeller, G., Fryer, C. L., Melia, F., \& Wang, Q. D. 2005, "Diffuse X-Rays from the Arches and Quintuplet Clusters" Astrophys. J. , 623, 171

Rockefeller, G., Fryer, C. L., \& Melia, F. 2005, "Spin-induced Disk Precession in Sagittarius A*" Astrophys. J. , 635, 336

Röpke, F. K., Hillebrandt, W., Niemeyer, J. C., \& Woosley, S. E. 2006, "Multi-spot Ignition in Type Ia Supernova Models", Astron. and Ap., 448, 1 - 14 
Röpke, F. K., Woosley, S. E., \& Hillebrandt, W. 2007, Astrophys. J. , "Off-Center Ignition in Type Ia Supernovae. I. Initial Evolution and Implications for Delayed Detonation", $660,1344-1356$

Röpke, F. K., \& Woosley, S. E. 2006, "Surface detonation in type Ia supernova explosions?", Journal of Physics Conference Series, 46, 413 - 417

Rogers, T. M., Glatzmaier, G. A., \& Woosley, S. E. 2003, "Simulations of Two-Dimensional Turbulent Convection in a Density-Stratified Fluid", Phys. Rev. E, 67, 26315

Rogers, T.M. and Glatzmaier, G.A. 2005, "Penetrative Convection Within the Anelastic Approximation", Astrophys. J. , 620, 432-441

Scannapieco, E., Madau, P., Woosley, S., Heger, A. and Ferrara, A. 2005, "The Detectability of Pair-Production Supernovae at z ; 6", Astrophys. J. , 633, 1031 - 1041

Scannapieco, E., Ferrara, A., Heger, A., Madau, P., Schneider, R., \& Woosley, S. 2006, "Detecting Primordial Stars", New Astronomy Review, 50, 89 - 93

Socrates, A., Blaes, O., Hungerford, A.L., Fryer, C.L., "The Neutrino Bubble Instability: A Mechanism for Generating Pulsar Kicks", Astrophys. J. , 632, 531 - 562

Strack, P., \& Burrows, A. 2005, "Generalized Boltzmann Formalism for Oscillating Neutrinos", Phys. Rev. D, 71, 093004

Takahashi, K., Sato, K., Burrows, A., and Thompson, T.A. 2003, "Supernova Neutrinos, Neutrino Oscillations, and the Mass of the Progenitor Star," Phys. Rev. D, 68, 113009

Thompson, T. A., Burrows, A., \& Meyer, B. S. 2001, "The Physics of Proto-Neutron Star Winds: Implications for r-Process Nucleosynthesis", Astrophys. J. , 562, 887

Thompson, T. A., Burrows, A., \& Pinto, P. A. 2003, "Shock Breakout in Core-Collapse Supernovae and its Neutrino Signature", ApJ, 592, 434 - 456

Thompson, T. A., Quataert, E., \& Burrows, A. 2005, "Viscosity and Rotation in Core-Collapse Supernovae", Astrophys. J. , 620, 861 - 877

Walder, R., Burrows, A., Ott, C. D., Livne, E., Lichtenstadt, I., \& Jarrah, M. 2005, "Anisotropies in the Neutrino Fluxes and Heating Profiles in Two-dimensional, Time-dependent, Multigroup Radiation Hydrodynamics Simulations of Rotating Core-Collapse Supernovae" Astrophys. J. , 626, 317 - 332

Willems, et al. 2005, " Understanding Compact Object Formation and Natal Kicks. I. Calculation Methods and the Case of GRO J1655-40, Astrophys. J. , 625, 324 
Woosley, S. E., Heger, A., \& Weaver, T. A. 2002, "The Evolution and Explosion of Massive Stars, Reviews of Modern Physics, 74, 1015

Woosley, S. E., Wunsch, S., \& Kuhlen, M. 2003, "Carbon Ignition in Type Ia Supernovae: I. An Analytic Model", ApJ, 607, 921 - 930

Woosley, S. E., Heger, A., Cumming, A., Hoffman, R. D., Pruet, J., Rauscher, T., Schatz, H. Brown, B. A., Wiescher, M., \& Fisker, J. 2004, "Models for Type I X-Ray Bursts with Improved Nuclear Physics" Astrophys. J. Suppl. Ser. , 151, 75 - 102

Woosley, S. E., \& Heger, A. 2005, "The Progenitor Stars of Gamma-Ray Bursts", Astrophys. J. , 637, $914-921$

Woosley, S. E., \& Bloom, J. S. 2006, "The Supernova Gamma-Ray Burst Connection" Ann. Rev. Astron. Ap., 44, 507 - 556

Woosley, S. E., et al. 2007, "Type Ia supernovae”, Journal of Physics Conference Series, 78, $2081-2090$

Woosley, S. E., \& Heger, A. 2007, Nucleosynthesis and Remnants in Massive Stars of Solar Metallicity", Physics Reports, 442, 269

Woosley, S. E., Kasen, D., Blinnikov, S., \& Sorokina, E. 2007, "Type Ia Supernova Light Curves", Astrophys. J. , 662, 487 - 503

Wunsch, S., and Woosley, S. E. 2004 "Convection and Off-Center Ignition in Type Ia Supernovae", Astrophys. J. , 616, 1102

Young, P. A., Meakin, C., Arnett, D., \& Fryer, C.L. 2005, "The Impact of Hydrodynamic Mixing on Supernova Progenitors", Astrophys. J. , 629, L101

Young, P. A., et al. 2006, "Constraints on the Progenitor of Cassiopeia A", Astrophys. J. , $640,891-900$

Zhang, W., Woosley, S. E., \& MacFadyen, A. I. 2003, "Relativistic Jets in Collapsars", Astrophys. J. , 586, 356

Zhang, W., Woosley, S. E., \& Heger, A. 2003, "The Propagation and Eruption of Relativistic Jets from the Stellar Progenitors of Gamma-Ray Burstss", ApJ, 608, 365 - 377

Zhang, W. and MacFadyen, A. I. 2005, "RAM a Relativistic Adaptive Mesh Refinement Hydrodynamics Code", Astrophys. J. Suppl. Ser. , 164, 255 - 279

Zingale, M., Dursi, L. J., ZuHone, J., Calder, A. C., Fryxell, B., Plewa, T., Truran, J. W., Caceres, A., Olson, K., Ricker, P. M., Riley, K., Rosner, R., Siegel, A., Timmes, F. X.,\& Vladimirova, N. 2002, "Mapping Initial Hydrostatic Models in Godunov Codes", ApJS, 143, 539. 
Zingale, M., Woosley, S. E., Rendleman, C. A., Day, M. S., \& Bell, J. B. 2005, "ThreeDimensional Numerical Simulations of Rayleigh-Taylor Unstable Flames in Type Ia Supernovae", Astrophys. J. , 632, 1021 - 1034

Zingale, M., Woosley, S. E., Bell, J. B., Day, M. S., \& Rendleman, C. A. 2005, "The physics of flames in Type Ia supernovae", Journal of Physics Conference Series, 16, 405

Zingale, M., Almgren, A. S., Bell, J. B., Day, M. S., Rendleman, C. A., \& Woosley, S. E. 2006, Journal of Physics Conference Series, 46, 385 


\section{Conference Proceedings}

Alexakis, A. et al. 2003, "Shear mixing in classical Novae", AAS/High Energy Astrophysics Division, 35, 18.08 .

Blaes, O. M., Fryer, C. L., Socrates, A., "Neutrino-Driven Acoustic Wave Instabilities in Proto-Neutron Stars and Gamma-Ray Burst Accretion Flows", BAAS, 203, 5312

Blinnikov, S. I., Nadyozhin, D. K., Woosley, S. E., \& Sorokina, E. I. 2002, "Shock breakouts in $\mathrm{SNe} \mathrm{Ib} / \mathrm{c}$ ", in Nuclear Astrophysics, in Proceedings of the 11th Workshop on "Nuclear Astrophysics", Ringberg Castle, Tegernsee, Germany, February 11-16, 2002, p. 144.

Blinnikov, S., Chugai, N., Lundqvist, P., Nadyozhin, D., Woosley, S., \& Sorokina, E. 2003, "Observable Effects of Shocks in Compact and Extended Presupernovae" in From Twilight to Highlight: The Physics of Supernovae. Proceedings of the ESO/MPA/MPE Workshop held in Garching, Germany, 29-31 July 2002, p. 23.

Burrows, A., \& Thompson, T. A., 2003, "The Mechanism of Core-Collapse Supernova Explosions: A Status Report," in the proceedings of the ESO/MPA/MPE Workshop, From Twilight to Highlight: The Physics of Supernovae, held in Garching bei München, Germany, July 29-31, 2002, eds. Bruno Leibundgut and Wolfgang Hillebrandt (Springer-Verlag), p. 53.

Burrows, A., \& Thompson, T. A. 2003, "Neutrino-Matter Interaction Rates in Supernovae: The Essential Microphysics of Core Collapse," to be published in Core Collapse of Massive Stars, ed. C. Fryer (Kluwer Academic Press), 2003.

Burrows, A., Ott, C.D., \& Meakin, C. 2003, "Topics in Core-Collapse Supernova Theory," to be published in the proceedings of " 3 -D Signatures in Stellar Explosions: A Workshop honoring J. Craig Wheeler's 60th birthday," held June 10-13, 2003, Austin, Texas, USA

Burrows, A., Ott, C. D., \& Meakin, C. 2004, "Topics in core-collapse supernova-theory", in Cosmic explosions in three dimensions, ed. P Höflich, CUP, 209

Burrows, A. 2004, "Understanding Core-Collapse Supernovae", in the proceedings of the Twelfth Workshop on "Nuclear Astrophysics," a Tribute to an Explosive Astrophysicist, Wolfgang Hillebrandt, on the occasion of his 60th Birthday, held at the Ringberg Castle, Lake Tegernsee, Germany, astro-ph/0405427

Burrows, A. 2005, "Multi-Dimensional Simulations of Core-Collapse Supernovae: The Road to the Mechanism of Explosion", KITP: Astrophysics Seminars 
Burrows, A., Walder, R., Ott, C. D., \& Livne, E. 2005, "Rotating Core Collapse and Bipolar Supernova Explosions", in The Fate of the Most Massive Stars, eds. R. Humphreys and K. Stanek, San Francisco: Astronomical Society of the Pacific, 332, 350

Burrows, A. 2005, "Core-Collapse Supernova Theory, Rotation, and Quasi-Bipolar Explosions", in 1604 - 2004: Supernovae as Cosmological Lighthouses, eds. M. Turatto et al., San Francisco: Astronomical Society of the Pacific, 342, 184

Burrows, A., Dessart, L., Livne, E., \& Ott, C. D. 2006, "New ideas in the theory of core-collapse supernova explosions", in Nuclei in the Cosmos - IX, 25-30 June 2006, CERN., p.32.1

Burrows, A. 2006, "Recent Theoretical Results on Core-Collapse SNe" at KITP Conference: "Supernova and Gamma-Ray Burst Remnants"

Calder, A. C. et al. 2003, "Progress in Modeling Classical Nova Outbursts", AAS/High Energy Astrophysics Division, 35, 18.02

Chandra, S., Sinha, S., Parashar, M., Zhang, Y., Yang, J., \& Hariri, S. 2002, "Adaptive Runtime Management of SAMR Applications," 9th International Conference on High Performance Computing, December 2002.

Church, C. M., Heger, A., \& Woosley, S. 2006, "Multidimensional Simulations of Mixing in Zero-Metallicity Supernovae", Bulletin of the American Astronomical Society, 38, 1102

Dobbs-Dixon, I., \& Woosley, S. 2004, "Semiconvection in Massive Stars", Bulletin of the American Astronomical Society, 36, 785

Dursi, L. J. et al. 2003, "Microphysics of Astrophysical Flames", AAS/High Energy Astrophysics Division, 35, 18.03.

Dursi, L. J. \& Zingale, M. 2005, "Efficiency Gains from Time Refinement on Spatially Refined Block-Structured Meshes", in Adaptive Mesh Refinement-Theory and Applications - Proceedings of the Chicago Workshop on Adaptive Mesh Refinement Methods, Lecture Notes in Computational Science and Engineering, Vol. 41 eds. T. Plewa and T. Linde, 554, astro-ph/0310891

Dursi, L. J., \& Zingale, M. 2006, Bulletin of the American Astronomical Society, "Propagation of the First Flames in Type Ia Supernovae", 38, 1101

Dwivedula, M., Hariri, S., Parashar, M. 2002, "A software design model for parallel applications on heterogeneous systems", International Parallel and Distributed Processing Symposium: IPDPS 2002 Workshops 
Feng, W., Warren, M., Weigle, \& E. "Honey, I Shrunk the Beowulf," Proceedings of the 2002 International Conference on Parallel Processing, (2002)

Feng, W., Warren, M.\& Weigle, E., "The Bladed Beowulf: A Cost-Effective Alternative to Traditional Beowulfs," Proceedings of IEEE Cluster 2002, (2002)

Fryer, C. L. "Comparing GRB Progenitors", GRBs and Afterglow Astronomy, AIP conference proceedings, 662, 199

Fryer, C. L., Holz, D. E., Hughes, S. A., "Stellar Collapse and Gravitational Waves", 2003, Contributed to "Stellar Collapse", Kluwer-Dordrecht Publishers, ed. C. L. Fryer

Fryer, C.L. 2003, "Black Hole Formation from Stellar Collapse", Classical and Quantum Gravity, 20, 73

Fryer, C.L., Warren, M.S., Holz, D.E., Hughes, S.A., \& Dupuis, R. 2003 "Gravitational Waves from Core-Collapse", in SPIE conference proceedings, Kona coast, Hawaii

Fryer, C. L. 2003, "Explosions from Stellar Collapse", in "A Massive Star Odyssey, from Main Sequence to Supernova", Proceedings of IAU symposium No. 212, eds. K. van der Hucht, A. Herrero, \& C. Esteban.

Fryer, C.L. 2003, "Black Hole Formation from Stellar Collapse", Classical and Quantum Gravity, in press

Fryer, C. L., Warren, M. S., \& Hungerford, A. L. 2002, "Asymmetric supernovae" , in Nuclear Astrophysics, in Proceedings of the 11th Workshop on "Nuclear Astrophysics", Ringberg Castle, Tegernsee, Germany, February 11-16, 2002, p. 132.

Fryer, C. L., "Stellar Collapse", Kluwer-Dordrecht Publishers

Fryer, C. L., Dupuis, R., "Stellar Collapse and the Formation of Black Holes", proceedings for the conference on GRBS, Santa Fe, 2003, eds. Galassi, Kippen, \& Fenimore

Fryer, C. L., \& Hungerford, A. 2004, "Changing the r-Process Paradigm: Multi-Dimensional and Fallback Effects", in The r-Process: The Astrophysical Origin of the Heavy Elements and Related Rare Isotope Accelerator Physics, ed. Yong-Zhong Qian, World Scientific Publishing Company, Singapore, p, 234

Fryer, C. 2004, "Explosions from stellar Collapse", APS Meeting Abstracts, Denver, 5001

Fryer, C. L. 2004, "Stellar Collapse", Astrophysics and Space Science Library, Kluwer, 302

Fryer, C. L., Rockefeller, G., Timmes, F. X., Hungerford, A. L., \& Belle, K. E. 2005, "Open Issues in Core-Collapse Supernovae - Progenitors and 3-Dimensional Simulations", in Open Issues in Core Collapse Supernova Theory, eds. Anthony Mezzacappa and George M. Fuller, World Scientific Publishing Co., London, p. 136 
Fryer, C. L., \& Hungerford, A. 2005, "Neutron Star Formation", NATO ASIB Proc. 210: The Electromagnetic Spectrum of Neutron Stars, ed. A. Baykal et al., p. 3

Fryer, C. L. 2006, "Radiation Hydrodynamics in Astrophysics", in Computational Methods in Transport, ed. F. Graziani, Springer, Berlin, P. 3

Fryer, C. 2006, "The Supernova/gamma-ray Burst Connection from the Eyes of a Mechanism Theorist", KITP: Blackboard Lunch Series,

Glatzmaier, G. A. 2004, "Planetary and stellar dynamos: Challenges for next generation models", in Astrophysical and Geophysical Fluid Dynamics and Dynamos, eds. A.M. Soward, C.A. Jones, D.W. hughes, N.O. Weiss, in press.

Glatzmaier, G.A. 2005, "Planetary and Stellar Dynamos: Challenges for Next Generation Models", chapter 11 in "Fluid dynamics and dynamos in astrophysics and geophysics" eds. A.M. Soward, C.A. Jones, D.W. Hughes and N.O. Wiess (CRC Press)

Glatzmaier, G.A., Ogden, D.E. \& Clune, T.L. 2004, "Modeling the Earth's Dynamo", Geophysical Monograph 150, IUGG Volume 19, 13-24.

Hariri, S. 2003, "Enabling Autonomic Grid Applications," to appear in Proceedings of the Autonomic Computing Workshop, June 2003

Hariri, S. 2003, "AUTONOMIA: An Autonomic Computing Environment," IEEE 22nd International Performance, Computing, and Communication Conference, April 2003.

Heger, A., Woosley, S. E., Fryer, C. L., \& Langer, N. 2003, "Massive Star Evolution Through the Ages", in From Twilight to Highlight: The Physics of Supernovae. Proceedings of the ESO/MPA/MPE Workshop held in Garching, Germany, 29-31 July 2002, p. 3.

Heger, A. \& Woosley, S. E. 2002, "The Beginning of Stellar Nucleosynthesis", in Proceedings of the 11th Workshop on "Nuclear Astrophysics", Ringberg Castle, Tegernsee, Germany, February 11-16, 2002, p. 8

Heger, A., Woosley, S. E., Langer, N., \& Spruit, H. 2003, "Presupernova Evolution of Rotating Massive Stars and the Rotation Rate of Pulsars", to appear in Proc. IAU 215 "Stellar Rotation", eds. A. Maeder, P. Eenens, astro-ph/0301374

Heger, A., Woosley, S. E., Langanke, K., Kolbe, E., Rauscher, T., \& Hoffman, R. D. 2003, "Nucleosynthesis of Heavy Elements in Massive Stars" in Proceedings of Nuclei in the Cosmos VII, Fuji-Yoshida, Japan, July 8-12, 2002, Nuclear Physics A, 718C, 159 .

Heger, A., \& Woosley, S. E. 2004, "The Evolution and Fate of the First Massive Stars", Bulletin of the American Astronomical Society, 36, 703 
Heger, A., Woosley, S. E., Langer, N., \& Spruit, H. C. 2004, "Presupernova Evolution of Rotating Massive Stars and the Rotation Rate of Pulsars", in Stellar Rotation, eds. Andre Maeder and Philippe Eenens. San Francisco: Astronomical Society of the Pacific, 215, 591

Heger, A., Woosley, S. E., \& Baraffe, I. 2005, "Final Stages of the Most Massive Stars", in The Fate of the Most Massive Stars, eds. R. Humphreys and K. Stanek. San Francisco: Astronomical Society of the Pacific, 332, 339

Heger, A., \& Woosley, S. 2005, "Nucleosynthesis of pair-instability supernovae", in From Lithium to Uranium: Elemental Tracers of Early Cosmic Evolution, Proceedings of the international Astronomical Union, CUP, 228, 297

Hoffman, R. D., Rauscher, T., Heger, A., \& Woosley, S. E. 2002, "New Results on Nucleosynthesis in Massive Stars; Nuclear Data Needs for Nucleosynthesis", proceedings of the International Conference on Nuclear Data for Science and Technology, held in Tsukuba, Japan, October 2001. Ed. K. Shibata. (Journal of Nuclear Science and Technology), Suppl. 2, Vol. 2, p. 512.

Hoffman, R., Pruet, J., Woosley, S. E., Janka, H.-T., \& Buras, R. 2006, "Nucleosynthesis in Early Proton-rich Supernova Winds", in Nuclei in the Cosmos IX, 25-30 June 2006, CERN., p.165

Hungerford, A., Fryer, C., \& Warren, M. 2002, "Gamma-Ray Lines From Asymmetric Supernovae", American Astronomical Society Meeting, 200, 32.06.

Hungerford, A. L., "Radioactive Decay in Core-Collapse Supernovae", 2003, Contributed to "Stellar Collapse", Kluwer-Dordrecht Publishers, ed. C. L. Fryer

Hungerford, A. L., \& Fryer, C. L. 2005, Open Issues in Supernova Lightcurves: Asymmetries and Nickel/Cobalt Decay", in Open Issues in Core Collapse Supernova Theory, eds. Anthony Mezzacappa and George M. Fuller, World Scientific Publishing, London, P. 441

Hungerford, A. L., Fryer, C. L., Socrates, A., \& Blaes, O. 2005, NATO ASIB Proc. 210: "Proto-Neutron Star Neutrino Emission", in NATO ASIB Proc. 210:The Electromagnetic Spectrum of Neutron Stars, ed. A. Baykal et al., 111

Kuhlen, M., Woosley, S. E., \& Glatzmaier, G. A. 2002, "3D Anelastic Simulation of Convection in Massive Stars", American Astronomical Society Meeting, 200, 93.01.

Kuhlen, M., Woosley, S. E., \& Glatzmaier, G. A. 2003, "3D Anelastic Simulations of Convection in Massive Stars", in 3D Stellar Evolution, Proceedings of Workshop, Livermore, CA, July 2002, ASP Conf. Ser., 293, 147-156. 
Lloyd-Ronning, N. M., Fryer, C. L., Ramirez-Ruiz, E. "What are Luminosity Indicators Telling Us?", GRBs and Afterglow Astronomy, AIP conference proceedings, 662, 454

Ma, H., Woosley, S., Kuhlen, M., Evonuk, M., \& Glatzmaier, G. 2006, "Numerical Simulations of Carbon Ignition in Type Ia Supernovae", Bulletin of the American Astronomical Society, 38, 1178

Murphy, J. W., Burrows, A., \& Heger, A. 2004, "Pulsar Kicks and a Pulsational Analysis of the Cores of Massive Stars", Bulletin of the American Astronomical Society, 36, 688

Parashar, M., \& Hariri, S. 2002, "PRAGA: An Infrastructure for Runtime Management of Grid Applications.", in Proceedings of the NSF Next Generation Systems Program Workshop, IEEE/ACM Int. Parallel and Distributed Processing Symposium, Fort Lauderdate, Fl. CDROM, IEEE Computer Society Press, April 2002.

Rauscher, T., Heger, A., Hoffman, R. D., \& Woosley, S. E. 2002, "Nuclear Aspects of the s- and n-Processes in Massive Stars", to be published in Proc. 9th Int. Seminar on Interactions of Neutrons with Nuclei (ISINN-9), Dubna, Russia, May 2001.

Pruet, J. 2004, "Are Collapsars Responsible for Some r-Process Elements? How Could We Tell?", in The r-Process: The Astrophysical Origin of the Heavy Elements and Related Rare Isotope Accelerator Physics, ed. Yong-Zhong Qian et al., World Scientific Publishing Company, p. 214

Rogers, T., Glatzmaier, G., \& Woosley, S. E. 2003, "A New Model For Penetrative Convection in the Anelastic Approximation", in 3D Stellar Evolution, Procedings of meeting at LLNL, July, 2002,ASP Conf. Proc. 293, 34 - 40

Rogers, T. M., Glatzmaier, G. A., \& Woosley, S. E. 2002, "Simulations of 2D Turbulence in the Anelastic Approximation", American Astronomical Society Meeting, 200, 59.01

Warren, M. S., Weigle, E.H., and Feng, W., "High-Density Computing: A 240-Processor Beowulf in One Cubic Meter", Proceedings of SC 2002, IEEE Computer Society (2002)

Woosley, S. E., \& Heger, A. 2003, "Supernovae, Gamma-Ray Bursts, and Stellar Rotation", to appear in Proc. IAU 215 "Stellar Rotation", eds. A. Maeder, P. Eenens, astro-ph/0301373

Woosley, S. E., et al. 2003, "Nuclear Data Need for the Study of Nucleosynthesis in Massive Stars", in proceedings of Nuclei in the Cosmos VII, in press.

Woosley, S. E., Zhang, W., \& Heger, A. 2003, "Gamma-Ray Bursts and Jet-Powered Supernovae", in From Twilight to Highlight: The Physics of Supernovae, Proceedings of the ESO/MPA/MPE Workshop held in Garching, Germany, 29-31 July 2002, p. 87. 
Woosley, S. E., Zhang, W., \& Heger, A. 2003, "The Central Engines of Gamma-Ray Bursts", in Gamma-Ray Burst and Afterglow Astronomy, AIP Conf. Proc. 662, 185 $-192$

Woosley, S. 2004, "How Zero-metallicity Massive Stars End Their Life", KITP Conference: Galaxy-Intergalactic Medium Interactions

Woosley, S. E., Zhang, W., \& Heger, A. 2004, he Collapsar Model for Gamma-Ray Bursts", in Gamma-Ray Bursts: 30 Years of Discovery, AIP Conf Series, 727, 343

Woosley, S. E., \& Heger, A. 2004,"S upernovae, Gamma-Ray Bursts and Stellar Rotation", in Stellar Rotation, eds. Andre Maeder and Philippe Eenens. San Francisco: Astronomical Society of the Pacific, 215, 601

Woosley, S. E., Zhang, W., \& Heger, A. A. 2004, "The Collapsar Model, Supernovae, and X-Ray Flashes", ed. M. Feroci et al., Astronomical Society of the Pacific Conference Series, 312,327

Woosley, S. E. and Heger, A. 2005, "Supernovae, Gamma-Ray Bursts, and Stellar Rotation", in The Fate of the Most Massive Stars, ASP Conference Series, Vol. 332, ed. R. Humphreys and K. Stanek. Astronomical Society of the Pacific, p.407 - 417

Yang, J., Zhang, Y., and Hariri, S. 2003, "Autonomic Runtime Partitioning of SAMR applications." Submitted to IEEE Transactions on Parallel and Distributed Systems

Young, P. A., \& Fryer, C. L. 2006, "Uncertainties in Supernova Yields", Bulletin of the American Astronomical Society, 38, 1098

Zhang, W. \& Woosley, S. 2002, "Relativistic Jets in Collapsars" American Astronomical Society Meeting, 200, 32.13.

Zhang, W., \& Woosley, S. E. 2003, "Relativistic Jets from Collapsars: Gamma-Ray Bursts", in 3D Stellar Evolution, Proceedings of Workshop, Livermore, CA, July 2002, ASP Conf. Proc. 293, 321 - 328

Zhang, W., Woosley, S. E., MacFadyen, A. I., \& Heger, A. 2003, "Numerical Simulation of Relativistic Jets in Collapsars", AAS Meeting 23, paper 132.4, Bulletin of the American Astronomical Society, 35, No. 5, 1418.

Zhang, W., Woosley, S. E., MacFadyen, A. I. 2003, "Relativistic Jets in Collapsars", in Gamma-Ray Bursts and Afterglow Astronomy, AIP Conf. Proc. 662, 226

Zhang, W., and Fryer, C. L., "The Collapsar Engine for GRBs and Hypernovae", 2003, Contributed to "Stellar Collapse", Kluwer-Dordrecht Publishers, ed. C. L. Fryer 
Zhang, W., Woosley, S. E., \& Heger, A. 2004, "Numerical Simulations of Relativistic Jets in Collapsars", in Gamma-Ray Bursts: 30 Years of Discovery, AIP Conf. Series, 727, 376

Zhang, W., Woosley, S. E., \& MacFadyen, A. I. 2006, "Gamma-ray bursts: nature's brightest explosions", Journal of Physics Conference Series, 46, 403

Zhu, H., Parashar, M., Yang, J., Zhang, Z., Rao, S., \& Hariri, S. 2003, "Self-Adapting, Self-Optimizing Runtime Management of Grid Applications Using Pragma." in Proceedings of the NSF Next Generation Systems Program Workshop, IEEE/ACM Int. Parallel and Distributed Processing Symposium, April 2003.

Zingale, M. et al. 2002, "Investigations of Pointwise Ignition of Helium Deflagrations on Neutron Stars", American Astronomical Society Meeting, 200, 94.03.

Zingale, M., Woosley, S. E., Cumming, A., Calder, A., Dursi, L. J., Fryxell, B., Olson, K., Ricker, P., Rosner, R., \& Timmes, F. X. 2003, "Investigations of Pointwise Ignition of Helium Deflagrations on Neutron Stars", in 3D Stellar Evolution, Proceedings of the workshop, Livermore, July 2002, ASP Conf Proc. 293, 329 - 336

Zingale, M., Bell, J. B., Day, M. S., Rendleman, C. A., \& Woosley, S. E. 2003, "Direct Numerical Simulations of Flame Instabilities in Type Ia Supernovae", Bulletin of the American Astronomical Society, 35, 1279

Zingale, M., Woosley, S. E., Almgren, A. S., Bell, J. B., Day, M. S., \& Rendleman, C. A. 2005, "Turbulent Flames in SNe Ia", Bulletin of the American Astronomical Society, 37,467

This preprint was prepared with the AAS IATEX macros v4.0. 